\title{
Cyclopamine analogs bearing exocyclic methylenes are highly potent and acid-stable inhibitors of hedgehog signaling
}

\author{
Johann Moschner ${ }^{1}$, Anna Chentsova ${ }^{1}$, Nicole Eilert ${ }^{1}$, Irene Rovardi ${ }^{1}$, \\ Philipp Heretsch ${ }^{* 2}$ and Athanassios Giannis ${ }^{* 1}$
}

\section{Full Research Paper}

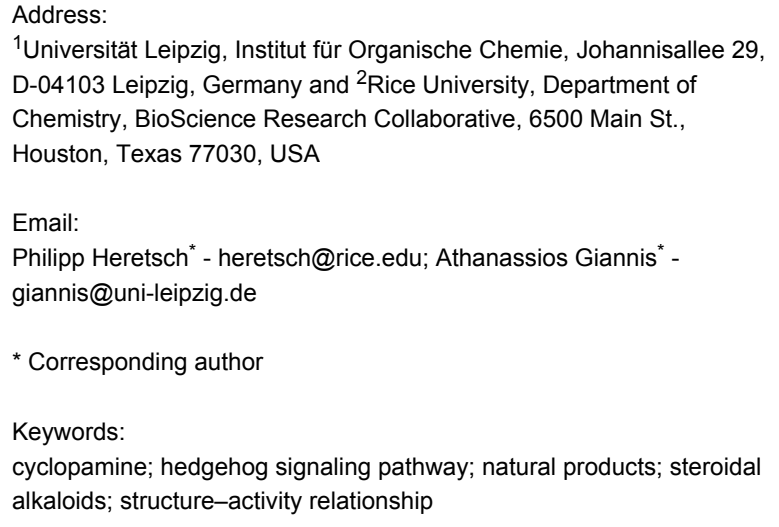

${ }^{1}$ Universität Leipzig, Institut für Organische Chemie, Johannisallee 29, D-04103 Leipzig, Germany and ${ }^{2}$ Rice University, Department of Chemistry, BioScience Research Collaborative, 6500 Main St., Houston, Texas 77030, USA

Email:

Philipp Heretsch* - heretsch@rice.edu; Athanassios Giannis * giannis@uni-leipzig.de

* Corresponding author

Keywords:

cyclopamine; hedgehog signaling pathway; natural products; steroidal alkaloids; structure-activity relationship

Beilstein J. Org. Chem. 2013, 9, 2328-2335

doi:10.3762/bjoc.9.267

Received: 16 August 2013

Accepted: 15 October 2013

Published: 31 October 2013

This article is part of the Thematic Series "Natural products in synthesis and biosynthesis".

Guest Editor: J. S. Dickschat

(C) 2013 Moschner et al; licensee Beilstein-Institut.

License and terms: see end of document.

\begin{abstract}
The chemical synthesis and biological evaluation of new cyclopamine analogs bearing exocyclic methylenes in different positions is described. Bis-exo-cyclopamine 6 was identified as a potent inhibitor of the Glil-dependent luciferase expression in ShhLIGHTII cells. An extension of this study to F-ring-modified structures shows the necessity of a rigidly positioned nitrogen atom for bioactivity as well as the presence of the $\mathrm{C} 21$ methyl group for acid stability and bioactivity.
\end{abstract}

\section{Introduction}

Hedgehog signaling is involved in embryonic development and plays an important role in the maintenance of stem cells, tissue repair and regeneration in adult organisms [1-4]. The erroneous activation of hedgehog signaling is tightly associated with the occurrence of basal cell carcinoma and medulloblastoma [5]. In addition, several other tumors are co-dependent on hedgehog signaling, examples for this type are cancers of the skin [6,7], brain [8,9], lung [10], pancreas [11], breast [12], prostate
$[13,14]$, colon [15], rhabdomyosarcoma [16], lymphoma [1719], multiple myeloma [17,20], and chronic myeloic leukemia [21-23]. More recently, other diseases like diabetes [24,25], neurodegenerative disorders [26], and trisomy [27,28], have been linked with hedgehog signaling.

Cyclopamine (1, see Figure 1) was the first inhibitor of the hedgehog signaling pathway to be identified. As a highly selec- 
tive inhibitor of the transmembrane protein Smoothened (Smo), an integral component of hedgehog signaling, it presents an attractive target for medicinal and pharmaceutical research $[29,30]$. Unfortunately, its direct development into a drug is hampered by its low metabolic stability (decomposition at $\mathrm{pH}<3)[31]$ and rather moderate potency $\left(\mathrm{IC}_{50} \sim 5 \mu \mathrm{M}\right)$.

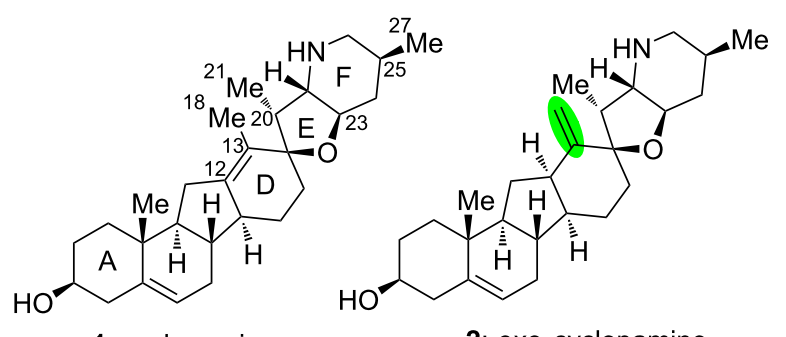

1: cyclopamine

2: exo-cyclopamine

Figure 1: Structures of cyclopamine and exo-cyclopamine.

We previously reported the first chemical synthesis of cyclopamine (1) starting from dehydroepiandrosterone and utilizing the $\mathrm{C}-\mathrm{H}$-functionalization logic and a biomimetic skeleton rearrangement [32,33]. Furthermore, quantum mechanical calculations guided our design and synthesis of exocyclopamine (2, see Figure 1), a ten-fold more potent and acidstable analog with an exo-methylene unit at C13-C18 [34]. Herein, we describe a comprehensive study of cyclopamine analogs bearing exo-methylene units in different positions and extent this rational to F-ring-modified structures.

\section{Results and Discussion}

Our synthetic approach to the analogs described here started from previously reported azide 3 which was converted in six steps [32] to the protected bis-exo-methylene compound 4. Carefully chosen conditions for the deprotection of the benzyl ether (DDQ, DCE/pH 7 phosphate buffer, $40{ }^{\circ} \mathrm{C}, 86 \%$ ) and the benzenesulfonylamine (sodium naphthalenide, DME, $-78{ }^{\circ} \mathrm{C}$, $79 \%$ ) allowed for the isolation of bis-exo-cyclopamine 6 in 18\% overall yield from 3 . The hydrogenation of intermediate 4 by using Wilkinson's catalyst in benzene yielded previously described exo-cyclopamine 2 and its C25 epimer 5. Deprotection with Raney-nickel (EtOH, $78{ }^{\circ} \mathrm{C}$ ) and then sodium naphthalenide (DME, $-78^{\circ} \mathrm{C}, 41 \%$ over two steps) furnished 25 -epiexo-cyclopamine 5 in 3\% overall yield from 3 .

A first set of exo-cyclopamine analogs with a modified F-ring was obtained starting again from azide 3 (see Scheme 1). Reduction of the lactone moiety to give a tetrahydrofuran was accomplished in three steps including (1) partial reduction to the lactol (DIBAl-H, THF, $-78{ }^{\circ} \mathrm{C}$ to $-60{ }^{\circ} \mathrm{C}, 95 \%$ ), (2) acetylation $\left(\mathrm{Ac}_{2} \mathrm{O}\right.$, pyridine, cat. DMAP, quant.) and (3) reductive removal of the so-obtained acetate $\left(\mathrm{Et}_{3} \mathrm{SiH}_{3} \mathrm{BF}_{3} \cdot \mathrm{Et}_{2} \mathrm{O},-78^{\circ} \mathrm{C}\right.$ to $-20{ }^{\circ} \mathrm{C}, 79 \%$ ). Removal of the benzyl ether, reduction of the azide moiety and concomitant alkylation was then all effected in one pot by using Raney-nickel in EtOH to give analog 8, an F-ring-opened exo-cyclopamine derivative in $27 \%$ overall yield from 3. Alternatively, the use of previously devised conditions for benzyl deprotection (DDQ, DCE/pH 7 phosphate buffer, $45{ }^{\circ} \mathrm{C}, 78 \%$ ) and then sodium borohydride-mediated reduction of the azide $\left(\mathrm{EtOH}, 65{ }^{\circ} \mathrm{C}, 62 \%\right)$ cleanly furnished primary amine 9 , an exo-cyclopamine derivative with no F-ring (35\% overall yield from 3 ).

A second set of F-ring-modified exo-cyclopamine derivatives was obtained starting from previously described 12-ß-triethylsilyloxy compound $\mathbf{1 0}$ (see Scheme 2) [32]. To construct the spirolactone 12, a three-step process consisting of (1) allylation (allylcerium chloride, THF, $0{ }^{\circ} \mathrm{C}, 93 \%$ ), (2) hydroboration/oxidation (9-BBN, THF, $70{ }^{\circ} \mathrm{C}$; then $\mathrm{NaBO}_{3}, \mathrm{H}_{2} \mathrm{O}, 50{ }^{\circ} \mathrm{C}, 91 \%$ ) and finally, (3) oxidative cyclization (BAIB, cat. TEMPO, $\mathrm{CH}_{2} \mathrm{Cl}_{2}, 25^{\circ} \mathrm{C}, 73 \%$ ) was employed. Deprotection of the silyl ether ( $\mathrm{HF}, \mathrm{MeCN}, 25^{\circ} \mathrm{C}, 87 \%$ ) and base-induced rearrangement of the steroid skeleton via the corresponding triflate ( $\mathrm{Tf}_{2} \mathrm{O}$, pyridine, $0{ }^{\circ} \mathrm{C} \rightarrow 50{ }^{\circ} \mathrm{C}, 46 \%$ ) gave $\mathrm{C}$-nor-D-homoderivative 13 which, in turn, was transformed into lactone azide 14 by using Evans' conditions (LDA, THF, $-78{ }^{\circ} \mathrm{C}$ to $-30{ }^{\circ} \mathrm{C}$, $10 \mathrm{~min}$; then trisylazide, THF, $-78^{\circ} \mathrm{C}, 1.5 \mathrm{~h}, 43 \%$ ) [35]. From 14 two additional exo-cyclopamine derivatives became accessible. Partial reduction to the lactol (DIBAl-H, THF, $-78{ }^{\circ} \mathrm{C}$ to $\left.-65{ }^{\circ} \mathrm{C}, 88 \%\right)$ and Horner-Wadsworth-Emmons reaction by using previously reported dimethyl \{3-[(4-methoxybenzyl)oxy]2-oxopropyl s phosphonate [32], $\left(\mathrm{Ba}(\mathrm{OH})_{2}, \mathrm{THF} / \mathrm{H}_{2} \mathrm{O}, 80{ }^{\circ} \mathrm{C}\right.$, $50 \%$ ) led to 15 which, in turn, was methylenated by employing the Peterson protocol $\left(\mathrm{TMSCH}_{2} \mathrm{CeCl}_{2}\right.$, THF, $-78{ }^{\circ} \mathrm{C}$; then TMEDA; then $\mathrm{HF}, \mathrm{MeCN}, 25{ }^{\circ} \mathrm{C}, 64 \%$ ) [36]. Towards this end the azide moiety was reduced and monoprotection of the so-obtained amine as a sulfonamide $\left(\mathrm{LiAlH}_{4}, \mathrm{THF}\right.$, $0{ }^{\circ} \mathrm{C} \rightarrow 25^{\circ} \mathrm{C}$; then benzenesulfonyl chloride, $\mathrm{Et}_{3} \mathrm{~N}$, DMF, $0{ }^{\circ} \mathrm{C}$, $69 \%$ ) gave 17. Deprotetion of the 4-methoxybenzyl ether (DDQ, DCE/pH 7 phosphate buffer, $25^{\circ} \mathrm{C}, 59 \%$ ) and cyclization under Mitsunobu conditions $\left(n-\mathrm{Bu}_{3} \mathrm{P}, \mathrm{DEAD}\right.$, toluene, $0{ }^{\circ} \mathrm{C} \rightarrow 25^{\circ} \mathrm{C}, 93 \%$ ) yielded piperidine 18. Deprotection of the benzyl ether by using previously devised conditions (DDQ, $\mathrm{DCE} / \mathrm{pH} 7$ phosphate buffer, $44{ }^{\circ} \mathrm{C}, 70 \%$ ) and the benzenesulfonylamine (sodium naphthalenide, $\mathrm{DME},-78{ }^{\circ} \mathrm{C}, 95 \%$ ) gave 20-demethyl-bis-exo-cyclopamine 19 in $7 \%$ overall yield starting from 14.

Starting from azido lactone $\mathbf{1 4}$ (see Scheme 2) a partial reduction to the lactol (DIBAl-H, THF, $-78{ }^{\circ} \mathrm{C}$ to $-65{ }^{\circ} \mathrm{C}, 88 \%$ ) and the Horner-Wadsworth-Emmons reaction by using triethyl phosphonoacetate $\left(\mathrm{Ba}(\mathrm{OH})_{2}, \mathrm{THF} / \mathrm{H}_{2} \mathrm{O}, 80^{\circ} \mathrm{C}, 55 \%\right)$ led to 


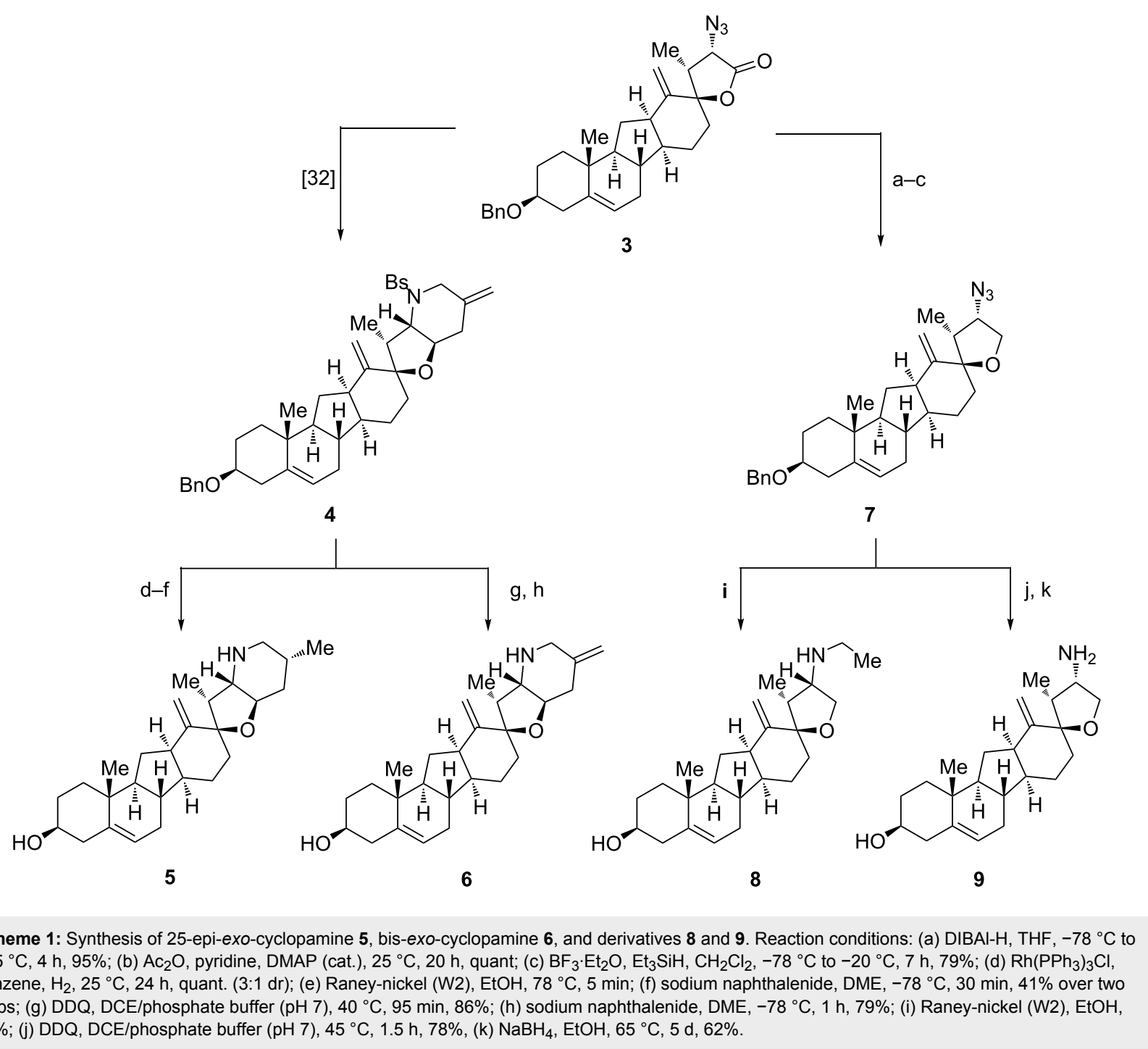

ethyl ester 20. Reduction of the azide moiety in $\mathbf{2 0}$ was carried out by using Staudinger's protocol $\left(\mathrm{Ph}_{3} \mathrm{P}, \mathrm{THF} / \mathrm{H}_{2} \mathrm{O}, 50{ }^{\circ} \mathrm{C}\right)$. Immediate protection of the obtained amine (benzenesulfonyl chloride, $\mathrm{Et}_{3} \mathrm{~N}, \mathrm{CH}_{2} \mathrm{Cl}_{2}, 40{ }^{\circ} \mathrm{C}, 97 \%$ over 2 steps) gave sulfonylamide 21. Reduction of the ester in $\mathbf{2 1}$ to the primary alcohol (DIBAl-H, THF, $-78{ }^{\circ} \mathrm{C}$ to $-40{ }^{\circ} \mathrm{C}, 97 \%$ ) and cyclization by employing Mitsunobu conditions $\left(n-\mathrm{Bu}_{3} \mathrm{P}, \mathrm{DEAD}\right.$, toluene, $0{ }^{\circ} \mathrm{C} \rightarrow 25{ }^{\circ} \mathrm{C}, 81 \%$ ) yielded pyrrolidine 22. Previously devised conditions for the deprotection (1. DDQ, DCE/pH 7 phosphate buffer, $40{ }^{\circ} \mathrm{C}$; 2. sodium naphthalenide, DME, $-78{ }^{\circ} \mathrm{C}, 46 \%$ ) finally afforded F-nor-20,25-bis-demethyl-exo-cyclopamine $\mathbf{2 3}$ in $9 \%$ overall yield starting from 14 .

Next, we examined all synthesized compounds for their ability to inhibit the Gli1-dependent luciferase expression in ShhLIGHTII cells, a clonal mouse fibroblast cell line which stably incorporates a Gli-dependent firefly luciferase reporter and a constitutive Renilla luciferase reporter [37]. The compounds were tested in a concentration range from $0.01 \mu \mathrm{M}$ to $10 \mu \mathrm{M}$. While analogs 8, 9, and 19 showed no activity (data not shown) in this concentration range, $\mathbf{5}, \mathbf{6}$, and $\mathbf{2 3}$ were active with 25-epi-exo-cyclopamine 5 having an $\mathrm{IC}_{50}$ of $3.29 \pm 0.31 \mu \mathrm{M}$, F-nor-20,25-bis-demethyl-exo-cyclopamine 23 of $6.4 \pm 0.9 \mu \mathrm{M}$, and bis-exo-cyclopamine 6 being the most active with an $\mathrm{IC}_{50}$ of $0.20 \pm 0.01 \mu \mathrm{M}$ (see Figure 2).

Given the negative test results of compounds 8 and 9 it becomes evident that the F-ring is necessary for bioactivity. The piperidine moiety provides a rather rigidly placed nitrogen atom. Nevertheless, a pyrrolidine as in compound $\mathbf{2 3}$ still provides the correct orientation of the nitrogen atom. Despite compounds 8 and 9 being inactive in the assay, both derivatives induced cytotoxicity in the concentration range tested. Very subtle changes of the conformation of the piperidine ring significantly change 


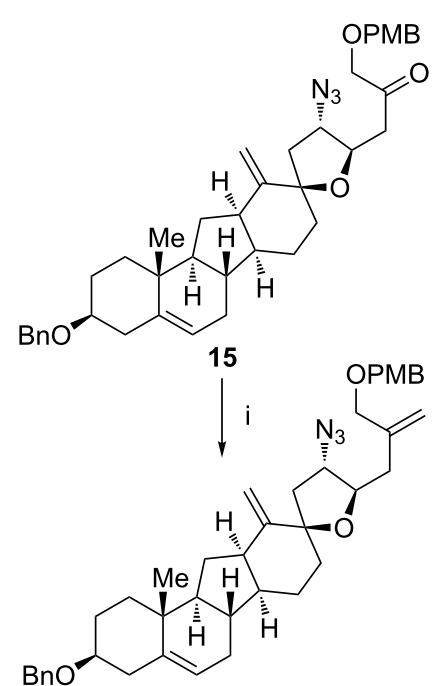

16

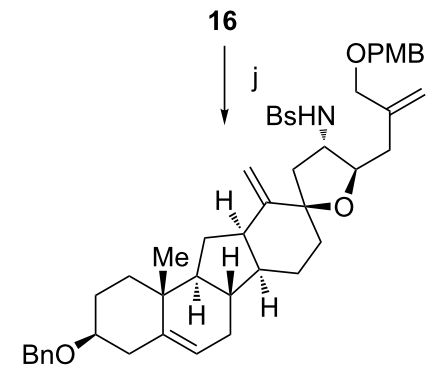

17

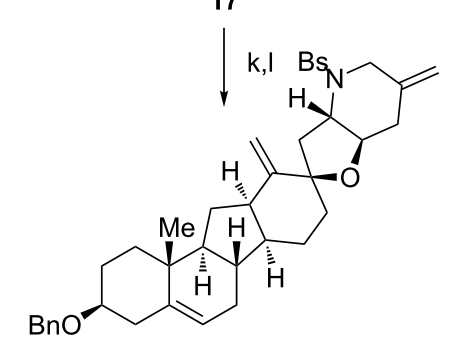

18

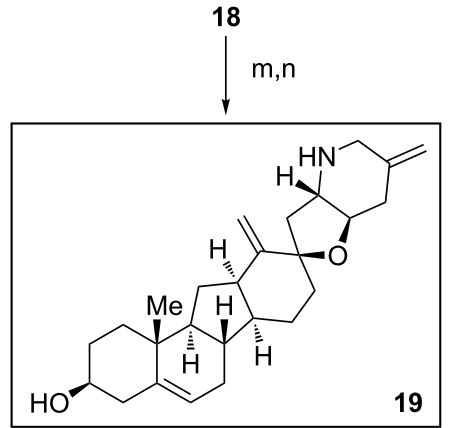

g,h

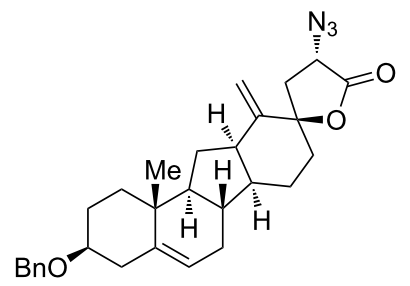

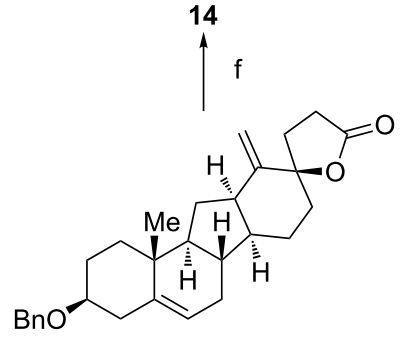

13

d,e

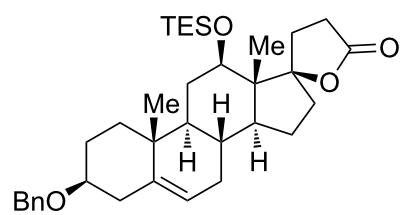

12 b,c

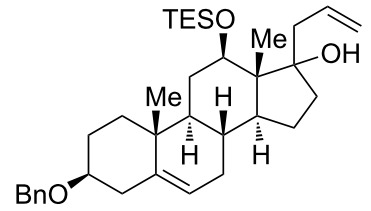

11

a

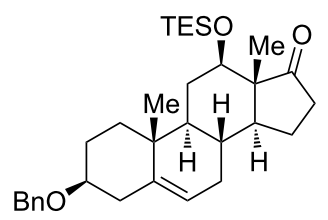

10 [32] g,o

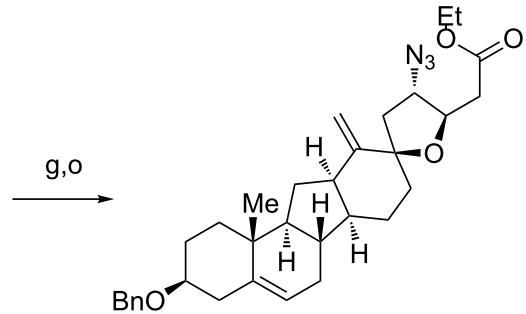

20

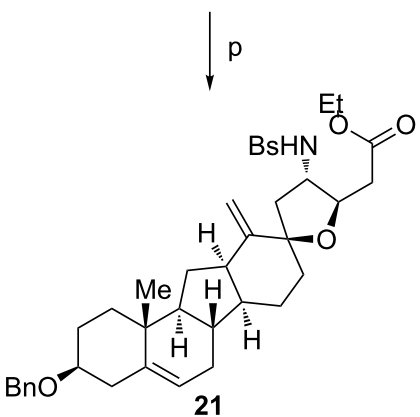

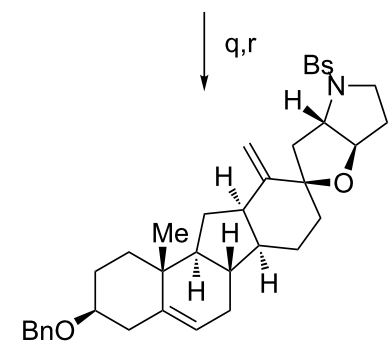

22 $\mathrm{s}, \mathrm{t}$

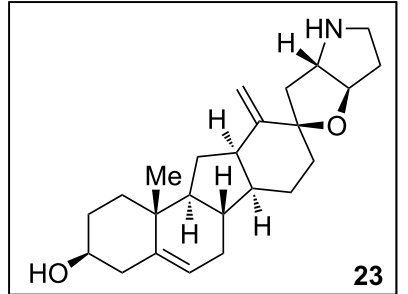

Scheme 2: Synthesis of 20-demethyl-bis-exo-cyclopamine 19 and F-nor-20,25-bis-demethyl-exo-cyclopamine 23. Reaction conditions: (a) allylcerium chloride, THF, $0{ }^{\circ} \mathrm{C}, 30 \mathrm{~min}, 93 \%$; (b) $9-\mathrm{BBN}$, THF, $70{ }^{\circ} \mathrm{C}, 6 \mathrm{~h}$; then $\mathrm{NaBO}_{3}, 50{ }^{\circ} \mathrm{C}, 12 \mathrm{~h}, 91 \%$; (c) $\mathrm{BAIB}, \mathrm{TEMPO}^{\circ} \mathrm{CH}_{2} \mathrm{Cl}_{2}, 25^{\circ} \mathrm{C}, 3 \mathrm{~h}, 73 \%$; (d) $\mathrm{HF} 50$ wt $\%$ in $\mathrm{H}_{2} \mathrm{O}, \mathrm{MeCN}, 20 \mathrm{~min}, 25^{\circ} \mathrm{C}, 87 \%$; (e) $\mathrm{Tf}_{2} \mathrm{O}$, pyridine, $0{ }^{\circ} \mathrm{C} \rightarrow 50{ }^{\circ} \mathrm{C}, 1.5 \mathrm{~h}$; then additional Tf $\mathrm{O}_{2} \mathrm{O}, 0{ }^{\circ} \mathrm{C} \rightarrow 50{ }^{\circ} \mathrm{C}, 2 \mathrm{~h}, 46 \%$; (f) $\mathrm{LDA}$, THF, $-78{ }^{\circ} \mathrm{C}$ to $-30{ }^{\circ} \mathrm{C}, 10 \mathrm{~min}$; then trisylazide, THF, $-78{ }^{\circ} \mathrm{C}, 1.5 \mathrm{~h}, 43 \%$; g) DIBAl-H, THF, $-78{ }^{\circ} \mathrm{C}$ to $-65^{\circ} \mathrm{C}, 2 \mathrm{~h}, 88 \%$; (h) dimethyl $(3-((4-$ methoxybenzyl)oxy)-2-oxopropyl)phosphonate, $\mathrm{Ba}(\mathrm{OH})_{2}, \mathrm{THF} / \mathrm{H}_{2} \mathrm{O}, 80^{\circ} \mathrm{C}, 13 \mathrm{~h}, 50 \%$; (i) (trimethylsilyl)methylcerium chloride, $\mathrm{THF},-78^{\circ} \mathrm{C}, 30 \mathrm{~min}$; then TMEDA, $-78{ }^{\circ} \mathrm{C}, 15 \mathrm{~min}$; then HF 50 wt \% in $\mathrm{H}_{2} \mathrm{O}$, MeCN, $25^{\circ} \mathrm{C}, 10 \mathrm{~min}, 64 \%$; (j) $\mathrm{LiAlH}_{4}, \mathrm{THF}^{\circ} 0^{\circ} \mathrm{C} \rightarrow 25^{\circ} \mathrm{C}, 13 \mathrm{~h}$; then benzenesulfonyl chloride, $\mathrm{Et}_{3} \mathrm{~N}, \mathrm{DMF}, 0{ }^{\circ} \mathrm{C}, 25 \mathrm{~min}, 69 \%$; (k) DDQ, $\mathrm{CH}_{2} \mathrm{Cl}_{2} / \mathrm{phosphate} \mathrm{buffer} \mathrm{(pH} \mathrm{7),} 25^{\circ} \mathrm{C}, 2 \mathrm{~h}, 59 \%$; (I) $n$-Bu $3 \mathrm{P}, \mathrm{DEAD}$, toluene, $0{ }^{\circ} \mathrm{C} \rightarrow 25^{\circ} \mathrm{C}, 12 \mathrm{~h}, 93 \%$; (m) DDQ, DCE/phosphate buffer ( $\mathrm{pH} 7$ ), $44{ }^{\circ} \mathrm{C}, 50 \mathrm{~min}, 70 \%$; (n) sodium naphthalenide, DME, $-78{ }^{\circ} \mathrm{C}, 30$ min, $95 \%$; (o) triethyl phosphonoacetate, $\mathrm{Ba}(\mathrm{OH})_{2}, \mathrm{THF}, 80^{\circ} \mathrm{C}, 12 \mathrm{~h}, 55 \%$; (p) $\mathrm{PPh}_{3}, \mathrm{THF} / \mathrm{H}_{2} \mathrm{O}, 50{ }^{\circ} \mathrm{C}, 24 \mathrm{~h}$; then benzenesulfonyl chloride, $\mathrm{Et}_{3} \mathrm{~N}, \mathrm{CH}_{2} \mathrm{Cl}_{2}, 40^{\circ} \mathrm{C}, 5 \mathrm{~h}, 97 \%$; (q) DIBAI-H, THF, $-78{ }^{\circ} \mathrm{C}$ to $-40{ }^{\circ} \mathrm{C}, 3 \mathrm{~h}, 97 \%$; (r) $n-\mathrm{Bu}_{3} \mathrm{P}, \mathrm{DEAD}$, toluene, $0{ }^{\circ} \mathrm{C} \rightarrow 25^{\circ} \mathrm{C}, 24 \mathrm{~h}, 81 \%$; (s) DDQ, DCE/phosphate buffer (pH 7), $40{ }^{\circ} \mathrm{C}, 30 \mathrm{~min}, 62 \%$; (t) sodium naphthalenide, DME, $-78^{\circ} \mathrm{C}, 40 \mathrm{~min}, 74 \%$. 

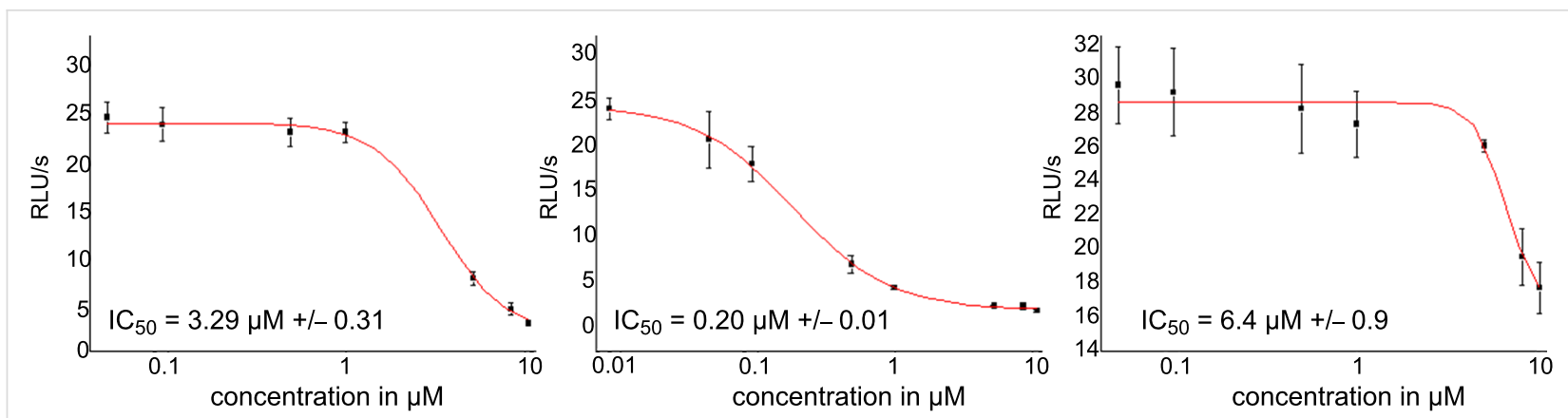

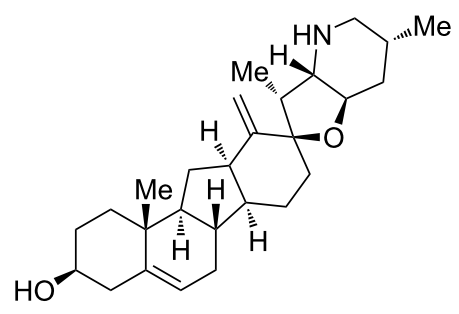

5: 25-epi-exo-cyclopamine

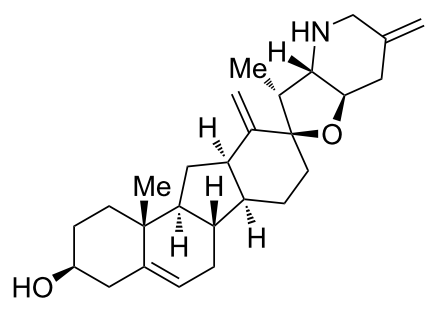

6: bis-exo-cyclopamine

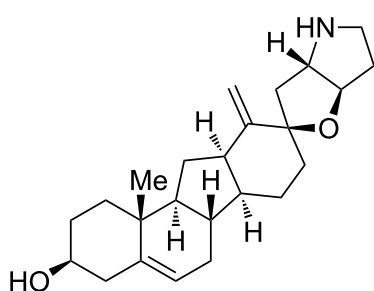

23: F-nor-20,25-bis-demethyl-exocyclopamine

Figure 2: $I_{50}$ values of Shh inhibition by compounds 5, 6 and 23 in a Gli1-reporter gene assay. Data were obtained from three independent experiments and represent the mean \pm standard deviation.

bioactivity: While 25-epi-exo-cyclopamine $\mathbf{5}$ shows reduced activity in comparison to exo-cyclopamine 2 , bis-exocyclopamine $\mathbf{6}$ is the most active compound tested in this study. Furthermore, the methyl group at C-20 seems to have a pronounced effect on the bioactivity, with 20-demethyl-bis-exocyclopamine 19 being completely inactive in the tested concentration range.

Finally, we studied the stability of all newly synthesized compounds towards acidic conditions. Therefore, they were exposed to a $\mathrm{pH}$ of approximately $1(\mathrm{MeOH}, 1 \mathrm{M} \mathrm{HCl})$ for $24 \mathrm{~h}$. After evaporation of all volatiles, ${ }^{1} \mathrm{H}$ NMR spectra were acquired and compared to the initially obtained spectra of the pure compounds. While compounds 5, 6, 8, and 9 remained unchanged, compounds 19 and 23 showed decomposition. This experiment emphasizes the importance of the C-21 methyl group for the stability of exo-cyclopamine derivatives. All synthesized compounds, the number of steps required, and the respective overall yield starting from 3 or $\mathbf{1 4}$, as well as their biological activity and stability under acidic conditions are summarized in Table 1.

\section{Conclusion}

In conclusion, we succeeded in identifying the new cyclopamine derivative bis-exo-cyclopamine 6 which surpasses the biological potency of the parent compound by the 25 -fold and is stable at $\mathrm{pH} 1$. Further insights were gained into the structure-activity relationship of F-ring-modified analogs of cyclopamine and the necessity of the C-21 methyl group for

Table 1: Synthesized exo-cyclopamine derivatives, number of steps, yields, results of their biological testing, and stability towards acid.

$\begin{array}{llll}\text { Compound } & \text { Number of steps } & \text { Overall } & \text { Potency in } \\ \text { from } 3 \text { or } 14 & \text { yield } & \text { Gli-assay }\end{array}$

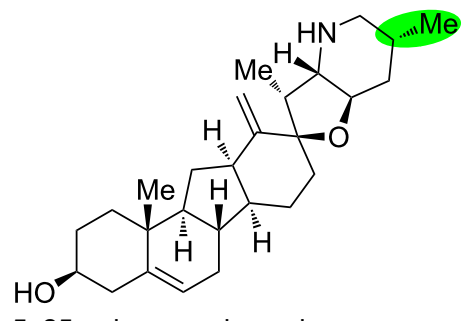

9 steps from $3 \quad 3 \% \quad 3.29 \pm 0.31 \mu \mathrm{M} \quad$ stable

5: 25-epi-exo-cyclopamine 
Table 1: Synthesized exo-cyclopamine derivatives, number of steps, yields, results of their biological testing, and stability towards acid. (continued)

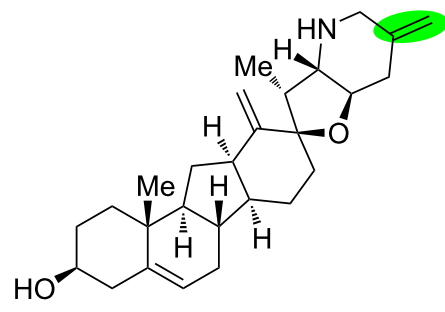

6: bis-exo-cyclopamine

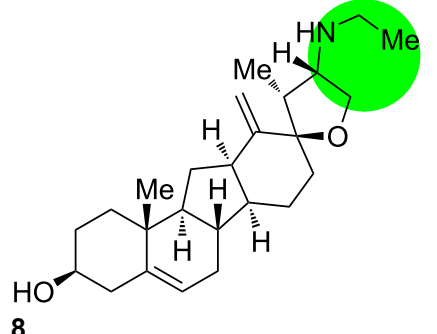

4 steps from 3

5 steps from 3 $35 \%$

8 steps from 14

$7 \%$

$>10 \mu \mathrm{M}$

decomp.
19: 20-demethyl-bis-exo-cyclopamine

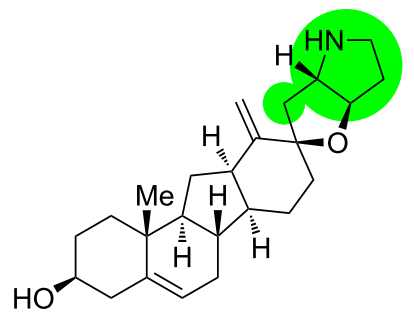

23: F-nor-20,25-bis-demethyl-exo-cyclopamine Previously synthesized and biologically evaluated:

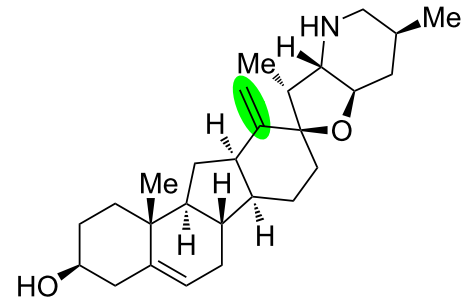

2: exo-cyclopamine
9 steps from $3 \quad 7 \% \quad 0.5 \mu \mathrm{M}[34] \quad$ stable 
Table 1: Synthesized exo-cyclopamine derivatives, number of steps, yields, results of their biological testing, and stability towards acid. (continued)<smiles>CC1=C2C[C@H]3[C@]4(C)CC[C@@H](O)CC4=CC[C@]3(C)[C@H]2CC[C@]12O[C@@H]1C[C@H](C)CN[C@H]1[C@H]2C</smiles>

10 steps from $3 \quad 5 \% \quad 5 \mu \mathrm{M}[34] \quad$ decomp.

1: cyclopamine

bioactivity and acid stability was revealed. Our designed analogs of cyclopamine are accessible in noticeably shorter and higher yielding synthetic routes than the parent compound, a fact that will further contribute to their usefulness in biological and medicinal studies.

\section{Supporting Information}

\section{Supporting Information File 1}

Experimental details and analytical data of all synthesized compounds are provided.

[http://www.beilstein-journals.org/bjoc/content/ supplementary/1860-5397-9-267-S1.pdf]

\section{Acknowledgements}

We thank Dr Lothar Hennig for recording NMR spectra and for his help in interpreting the 2D NMR spectra.

\section{References}

1. Keeler, R. F. Phytochemistry 1968, 7, 303. doi:10.1016/S0031-9422(00)86328-1

2. Keeler, R. F. Teratology 1970, 3, 169. doi:10.1002/tera.1420030209

3. Beachy, P. A.; Karhadkar, S. S.; Berman, D. M. Nature 2004, 432, 324. doi:10.1038/nature03100

4. Taipale, J.; Beachy, P. A. Nature 2001, 411, 349. doi:10.1038/35077219

5. Hidalgo, M.; Maitra, A. N. Engl. J. Med. 2009, 361, 2094. doi:10.1056/NEJMcibr0905857

6. Gailani, M. R.; Ståhle-Bäckdahl, M.; Leffell, D. J.; Glyn, M.; Zaphiropoulos, G.; Undén, A. B.; Dean, M.; Brash, D. E.; Bale, A. E.; Toftgård, R. Nat. Genet. 1996, 14, 78. doi:10.1038/ng0996-78

7. Hahn, H.; Wicking, C.; Zaphiropoulos, P. G.; Gailani, M. R.; Shanley, S.; Chidambaram, A.; Vorechovsky, I.; Holmberg, E.; Undén, A. B.; Gillies, S.; Negus, K.; Smyth, I.; Pressman, C.; Leffell, D. J.; Gerrard, B.; Goldstein, A. M.; Dean, D.; Toftgård, R.; Chenevix-Trench, G.; Wainwright, B.; Bale, A. E. Cell 1996, 85, 841. doi:10.1016/S0092-8674(00)81268-4

8. Zurawel, R. H.; Allen, C.; Chiappa, S.; Cato, W.; Biegel, J.; Cogen, P.; de Sauvage, F.; Raffel, C. Genes, Chromosomes Cancer 2000, 27, 44. doi:10.1002/(SICI)1098-2264(200001)27:1<44::AID-GCC6>3.0.CO;2-V
9. Bar, E. E.; Chaudhry, A.; Lin, A.; Fan, X.; Schreck, K.; Matsui, W.; Piccirillo, S.; Vescovi, A. L.; DiMeco, F.; Olivi, A.; Eberhart, C. G. Stem Cells 2007, 25, 2524. doi:10.1634/stemcells.2007-0166

10. Watkins, D. N.; Berman, D. M.; Baylin, S. B. Cell Cycle 2003, 2, 195. doi:10.4161/cc.2.3.378

11. Dosch, J. S.; di Magliano, M. P.; Simeone, D. M. Pancreatology 2010, 10, 151. doi:10.1159/000225923

12. Vořechovský, I.; Benediktsson, K. P.; Toftgård, R. Eur. J. Cancer 1999, 35, 711. doi:10.1016/S0959-8049(99)00017-9

13. Fan, L.; Pepicelli, C. V.; Dibble, C. C.; Catbagan, W.; Zarycki, J. L.; Laciak, R.; Gipp, J.; Shaw, A.; Lamm, M. L. G.; Munoz, A.; Lipinski, R.; Thrasher, J. B.; Bushman, W. Endocrinology 2004, 145, 3961. doi:10.1210/en.2004-0079

14. Karhadkar, S. S.; Bova, G. S.; Abdallah, N.; Dhara, S.; Gardner, D.; Maitra, A.; Isaacs, J. T.; Berman, D. M.; Beachy, P. A. Nature 2004, 431, 707. doi:10.1038/nature02962

15. Berman, D. M.; Karhadkar, S. S.; Maitra, A.; Montes de Oca, R.; Gerstenblith, M. R.; Briggs, K.; Parker, A. R.; Shimada, Y.; Eshleman, J. R.; Watkins, D. N.; Beachy, P. A. Nature 2003, 425, 846 doi:10.1038/nature01972

16. Teglund, S.; Toftgård, R. Biochim. Biophys. Acta, Rev. Cancer 2010, 1805, 181. doi:10.1016/j.bbcan.2010.01.003

17. Dierks, C.; Grbic, J.; Zirlik, K.; Beigi, R.; Englund, N. P.; Guo, G.-R.; Veelken, H.; Engelhardt, M.; Mertelsmann, R.; Kelleher, J. F.; Schultz, P.; Warmuth, M. Nat. Med. 2007, 13, 944. doi:10.1038/nm1614

18. Hegde, G. V.; Munger, C. M.; Emanuel, K.; Joshi, A. D.; Greiner, T. C.; Weisenburger, D. D.; Vose, J. M.; Joshi, S. S. Mol. Cancer Ther. 2008, 7, 1450. doi:10.1158/1535-7163.MCT-07-2118

19. Kawahara, T.; Kawaguchi-Ihara, N.; Okuhashi, Y.; Itoh, M.; Nara, N.; Tohda, S. Anticancer Res. 2009, 29, 4629.

20. Peacock, C. D.; Wang, Q.; Gesell, G. S.; Corcoran-Schwartz, I. M.; Jones, E.; Kim, J.; Devereux, W. L.; Rhodes, J. T.; Huff, C. A.; Beachy, P. A.; Watkins, D. N.; Matsui, W. Proc. Natl. Acad. Sci. U. S. A. 2007, 104, 4048. doi:10.1073/pnas.0611682104

21. Zhao, C.; Chen, A.; Jamieson, C. H.; Fereshteh, M.; Abrahamsson, A.; Blum, J.; Kwon, H. Y.; Kim, J.; Chute, J. P.; Rizzieri, D.; Munchhof, M.; VanArsdale, T.; Beachy, P. A.; Reya, T. Nature 2009, 458, 776. doi:10.1038/nature07737

22. Bai, L.-Y.; Chiu, C.-F.; Lin, C.-W.; Hsu, N.-Y.; Lin, C.-L.; Lo, W.-J.; Kao, M.-C. Leukemia 2008, 22, 226. doi:10.1038/sj.leu.2404978 23. Dierks, C.; Beigi, R.; Guo, G.-R.; Zirlik, K.; Stegert, M. R.; Manley, P.; Trussel, C.; Schmitt-Graeff, A.; Landwerlin, K.; Veelken, H.; Warmuth, M. Cancer Cell 2008, 14, 238. doi:10.1016/j.ccr.2008.08.003 24. Suh, J. M.; Gao, X.; McKay, J.; McKay, R.; Salo, Z.; Graff, J. M. Cell Metab. 2006, 3, 25. doi:10.1016/j.cmet.2005.11.012 
25. Teperino, R.; Amann, S.; Bayer, M.; McGee, S. L.; Loipetzberger, A.; Connor, T.; Jaeger, C.; Kammerer, B.; Winter, L.; Wiche, G.;

Dalgaard, K.; Selvaraj, M.; Gaster, M.; Lee-Young, R. S.;

Febbraio, M. A.; Knauf, C.; Cani, P. D.; Aberger, F.; Penninger, J. M.; Pospilik, J. A.; Esterbauer, H. Cell 2012, 151, 414.

doi:10.1016/j.cell.2012.09.021

26. Gonzalez-Reyes, L. E.; Verbitsky, M.; Blesa, J.; Jackson-Lewis, V.; Paredes, D.; Tillack, K.; Phani, S.; Kramer, E. R.; Przedborski, S.; Kottmann, A. H. Neuron 2012, 75, 306. doi:10.1016/j.neuron.2012.05.018

27. Nowaczyk, M. J.; Huggins, M. J.; Tomkins, D. J.; Rossi, E.; Ramsay, J. A.; Woulfe, J.; Scherer, S. W.; Belloni, E. Clin. Genet. 2000, 57, 388. doi:10.1034/j.1399-0004.2000.570510.x

28. Decker, S.; Zirlik, K.; Djebatchie, L.; Hartmann, D.; Ihorst, G.; Schmitt-Graeff, A.; Herchenbach, D.; Jumaa, H.; Warmuth, M.; Veelken, H.; Dierks, C. Blood 2012, 119, 997. doi:10.1182/blood-2011-06-359075

29. Heretsch, P.; Tzagkaroulaki, L.; Giannis, A. Bioorg. Med. Chem. 2010, 18, 6613. doi:10.1016/j.bmc.2010.07.038

30. Heretsch, P.; Tzagkaroulaki, L.; Giannis, A. Angew. Chem. 2010, 122, 3492. doi:10.1002/ange.200906967

31. Wilson, S. R.; Strand, M. F.; Krapp, A.; Rise, F.; Petersen, D.; Krauss, S. J. Pharm. Biomed. Anal. 2010, 52, 707. doi:10.1016/j.jpba.2010.02.017

32. Giannis, A.; Heretsch, P.; Sarli, V.; Stößel, A. Angew. Chem. 2009, 121, 8052. doi:10.1002/ange.200902520

33. Heretsch, P.; Rabe, S.; Giannis, A. J. Am. Chem. Soc. 2010, 132, 9968. doi:10.1021/ja103152k

34. Heretsch, P.; Büttner, A.; Tzagkaroulaki, L.; Zahn, S.; Kirchner, B.; Giannis, A. Chem. Commun. 2011, 47, 7362. doi:10.1039/C1CC11782C

35. Evans, D. A.; Britton, T. C. J. Am. Chem. Soc. 1987, 109, 6881. doi:10.1021/ja00256a069

36. Johnson, C. R.; Tait, B. D. J. Org. Chem. 1987, 52, 281. doi:10.1021/jo00378a024

37. Taipale, J.; Chen, J. K.; Cooper, M. K.; Wang, B.; Mann, R. K.; Milenkovic, L.; Scott, M. P.; Beachy, P. A. Nature 2000, 406, 1005. doi: $10.1038 / 35023008$

\section{License and Terms}

This is an Open Access article under the terms of the Creative Commons Attribution License (http://creativecommons.org/licenses/by/2.0), which permits unrestricted use, distribution, and reproduction in any medium, provided the original work is properly cited.

The license is subject to the Beilstein Journal of Organic Chemistry terms and conditions: (http://www.beilstein-journals.org/bjoc)

The definitive version of this article is the electronic one which can be found at: doi:10.3762/bjoc. 9.267 